\title{
Progresso genético e seleção precoce em genótipos de pinhão-manso em fase juvenil
}

\author{
Ana Paula Mora da SILVA, Liliana Rocivalda Gomes LEITÃO², \\ Linda Brenna Ribeiro ARAÚJO2*, Jonas CUNHA NETO², \\ Cândida Hermínia Campos de Magalhães BERTINI²
}

\author{
${ }^{1}$ Embrapa Agrossilvipastoril, Sinop, MT, Brasil. \\ 2Programa de Pós-Graduação em Agronomia (Fitotecnia), Universidade Federal do Ceará, Fortaleza, CE, Brasil. \\ ${ }^{3}$ Empresa FJ Consultoria, Sorriso, MT, Brasil. \\ *E-mail: lindabrenna@gmail.com
}

(ORCID: 0000-0001-6073-9243; 0000-0003-2667-0689; 0000-0002-3554-3908; 0000-0003-3190-9623; 0000-0003-2949-5660)

\begin{abstract}
Recebido em 05/01/2021; Aceito em 29/11/2021; Publicado em 17/12/2021.
RESUMO: O pinhão manso (Jatropha curcas L.) é uma euforbiácea utilizada na produção de biodiesel. A espécie possui base genética estreita o que dificulta o processo de lançamento de cultivares. Caracterizar precocemente os genótipos constitui etapa importante no melhoramento da cultura. Objetivou-se com este estudo realizar uma seleção precoce em caracteres morfoagronômicos, predizer o progresso genético de tais caracteres e indicar genitores potenciais para obtenção de progênies. O delineamento experimental utilizado foi o de blocos casualizados com 26 tratamentos e três repetições. As variáveis morfoagronômicas foram analisadas via modelos mistos e o progresso genético obtido pela seleção direta, indireta e índices de seleção. A dissimilaridade genética foi determinada pela distância de Mahalanobis, com agrupamento hierárquico UPGMA e coeficiente de correlação cofenética adquirido de 1.000 permutações. As estimativas demonstraram variabilidade genética, com identificação de genótipos juvenis promissores. Os genótipos JCCE034, JCCE014 e JCCE103 apresentam melhores progressos genéticos e os genótipos JCCE036 e JCCE86 apresentam maiores divergências genéticas, formando clusters individuais. Os genótipos de pinhão-manso são promissores na seleção precoce e possuem satisfatórios ganhos com a seleção para os caracteres avaliados. Os genótipos apresentam boa capacidade para compor grupos de genitores em cruzamentos direcionados, constituindo populações-base no melhoramento de J. curcas.
\end{abstract}

Palavras-chave: Jatropha curcas; diversidade genética; ganhos com a seleção; índices de seleção.

\section{Genetic progress and early selection of juvenile physic nut genotypes}

\begin{abstract}
Physic nut (Jatropha curcas L.) is a euphorbia used in the production of biodiesel. The species has a narrow genetic base which the process of launching cultivars is difficult. Early characterization of the genotypes is an important step in crop breending. The aims of this study were to perform an early selection in morpho-agronomic traits, to predict the genetic progress of such traits and to indicate potential parents for progeny development. The experimental design used for the randomized blocks with 26 treatments and three replications. The morpho-agronomic traits were analyzed via mix models and the genetic progress added by direct, indirect selection and selection indexes. The genetic dissimilarity was provided by the Mahalanobis distance, with UPGMA hierarchical grouping and co-phenetic correlation coefficient acquired from 1,000 permutations. The indicators demonstrated genetic variability, with the identification of promising juvenile genotypes. The genotypes JCCE034, JCCE014 and JCCE103 show better genetic progress and the genotypes JCCE036 and JCCE86 show greater genetic divergences, forming individual clusters. J. curcas genotypes are promising in early selection and have satisfactory genetics gains for the traits. The genotypes have a good ability to compose groups of parents in targeted crosses, constituting base populations in the improvement of J. curcas. Keywords: Jatropha curcas; genetic diversity; selection gains; selection indexes.
\end{abstract}

\section{INTRODUÇÃO}

O pinhão-manso (Jatropha curcas L.) é uma espécie da família Euphorbiaceae que responde bem as condições ambientais das regiões tropicais e subtropicais do planeta (AYIZANNON et al., 2017). A oleagionosa apresenta ampla diversidade de uso, mas se destaca no cenário energético global por ser fonte alternativa de combustíveis fósseis, sendo destinada à produção de biodiesel (MORAES et al., 2017).
A espécie se caracteriza por ser alógama, com poucas variações genéticas, uma vez que boa parte dos genótipos africanos e americanos utilizados são oriundos de seleções simples em populações locais (AYIZANNON et al., 2017). Essa estreita base genética dificulta o processo de melhoramento e lançamento de cultivares, por isso, coletas no centro de origem e diversidade da espécie vêm sendo feitas a fim de enriquecer os germoplasmas (MAVUSO et al., 
2016; LAVIOLA et al., 2019) e ampliar a diversidade da espécie.

Variações genéticas foram observadas em acessos brasileiros de pinhão-manso e contribuíram na caracterização de germoplasma e identificação de genótipos com caracteres favoráveis de J. curcas (DÍAS et al., 2017; SOUSA et al., 2019). Identificar a variabilidade genética do germoplasma é outra etapa importante nos programas de melhoramento da espécie com objetivo de realizar a seleção de genótipos (MORAES et al., 2017) para compor genitores em futuros cruzamentos direcionados.

$\mathrm{Na}$ seleção desses genitores, recomenda-se identificar as variáveis passíveis de seleção, sejam morfológicas, fisiológicas e/ou produtivas (FRANCIS et al., 2017), bem como identificar a magnitude e sentido das correlações entre os caracteres avaliados (ALVES et al., 2019). Posteriormente, pode-se realizar a seleção para múltiplos caracteres, considerando uma série de combinações entre as variáveis para a seleção conjunta das variáveis de interesse (CRUZ et al., 2012). O objetivo, além da seleção simultânea, é a predição dos ganhos genéticos nas gerações seguintes (AYIZANNON et al., 2017).

Estimar os parâmetros genéticos e correlações visando obter ganhos de seleção é de grande importância no melhoramento de pinhão-manso. O intuito é obter uma boa performance agronômica em genótipos avaliados ainda na fase juvenil para compor os genitores em hibridações direcionadas. Portanto, objetivou-se nesse trabalho realizar uma seleção precoce baseada em caracteres morfológicos e agronômicos e predizer progressos genéticos nos caracteres avaliados a fim de indicar genitores potenciais para obtenção de progênies a serem melhoradas.

\section{MATERIAL E MÉTODOS}

O ensaio foi realizado no município de Pentecoste-CE, em área experimental da Fazenda Vale do Curu, localizada nas coordenadas $3^{\circ} 47^{\prime} \mathrm{S}$ e $39^{\circ} 16^{\prime} \mathrm{W}$, com 45,0 m de altitude. O clima da área é classificado como tropical chuvoso (Aw'), conforme Köppen-Geiger, caracterizado com estação seca de cinco a oito meses, com médias anuais de $29{ }^{\circ} \mathrm{C}$ de temperatura, $59 \%$ de umidade relativa e $756 \mathrm{~mm}$ de precipitação.

Os dados climáticos do período experimental foram obtidos na estação meteorológica da própria fazenda. A adubação de cobertura foi realizada com base na caracterização física e química da análise de solo e nas recomendações para adubação de mamona para o Estado do Ceará, diante da escassez de informações de adubação para o pinhão-manso (AQUINO et al., 1993).

No experimento foram utilizados 26 genótipos de pinhão-manso provenientes do germoplasma da Universidade Federal do Ceará - UFC (Tabela 1). Os genótipos foram arranjados sob delineamento experimental de blocos casualizados completos, com três repetições e duas plantas por parcela espaçadas de $2 \times 3 \mathrm{~m}$. As avaliações morfológicas foram realizadas aos três, seis e nove meses após o plantio e as avaliações agronômicas foram realizadas aos 12 meses após o plantio.

$\mathrm{Na}$ caracterização morfológica foram avaliados os descritores: altura de plantas - AP (entre a base do caule e extremidade do ramo, em $\mathrm{cm}$ ); diâmetro do caule - DC (a uma distância de $\pm 7,5 \mathrm{~cm}$ da base do caule, em $\mathrm{mm}$ ); número de ramos totais - NRT; comprimento do entrenó do caule principal - CECP (média de cinco entrenós do caule principal, em $\mathrm{mm}$ ); comprimento do entrenó de ramos primários - CERP (média de cinco entrenós em três ramos primários, em $\mathrm{mm}$ ); comprimento do entrenó de ramos produtivos - CERI (média de cinco entrenós em três ramos com inflorescências, em $\mathrm{mm}$ ); e, porcentagem de ramos secundários - RS (relação entre número de ramos secundários e número total de ramos, em \%).

Tabela 1. Identificação e origem dos genótipos de pinhão-manso. Table 1. Identification and origin of physic nut genotypes.

\begin{tabular}{ccc}
\hline Tratamento & Identificação & Origem \\
\hline 1 & JCCE069 & Piauí \\
2 & JCCE070 & Piauí \\
3 & JCCE034 & Ceará \\
4 & JCCE035 & Ceará \\
5 & JCCE036 & Ceará \\
6 & JCCE037 & Ceará \\
7 & JCCE038 & Ceará \\
8 & JCCE043 & Ceará \\
9 & JCCE064 & Ceará \\
10 & JCCE053 & Ceará \\
11 & JCCE055 & Ceará \\
12 & JCCE056 & Ceará \\
13 & JCCE060 & Ceará \\
14 & JCCE061 & Ceará \\
15 & JCCE014 & Ceará \\
16 & JCCE020 & Ceará \\
17 & JCCE086 & Paraíba \\
18 & JCCE102 & Rio de Janeiro \\
19 & JCCE099 & Rio de Janeiro \\
20 & JCCE103 & Rio de Janeiro \\
21 & JCCE106 & Rio de Janeiro \\
22 & JCCE100 & Rio de Janeiro \\
23 & JCCE101 & Rio de Janeiro \\
24 & JCCE107 & Rio de Janeiro \\
25 & JCCE108 & Rio de Janeiro \\
26 & JCCE105 & Rio de Janeiro \\
\hline & &
\end{tabular}

$\mathrm{Na}$ caracterização agronômica os descritores avaliados foram: número de frutos por planta - NFP; número de sementes por planta - NSP; número de sementes por fruto NSF (relação entre NSP e NFP); produção por planta PROD (massa do total de sementes colhidas, em g); e, massa de 100 sementes - M100 (em g).

$\mathrm{Na}$ predição dos parâmetros genéticos e valores genotípicos dos caracteres, os dados foram submetidos às análises individuais de modelos mistos, via Restricted Maximum Likelihood - REML, considerando o modelo estatístico (Equação 1):

$$
y=\mathrm{Xr}+\mathrm{Zg}+\mathrm{Wp}+\mathrm{e}
$$

em que: $y=$ vetor de dados; $r=$ vetor dos efeitos de repetição (efeito fixo); $g$ = vetor dos genótipos (efeitos aleatórios); $p=$ vetor dos efeitos de parcela; $e=$ vetor de erros (efeitos aleatórios). As letras $\mathrm{X}, \mathrm{Z}$ e W se referem as matrizes de incidência para os tais efeitos.

$\mathrm{Na}$ análise conjunta foi considerado o seguinte modelo (Equação 2):

$$
y=\mathrm{Xr}+\mathrm{Zg}+\mathrm{Wp}+\mathrm{Ti}+\mathrm{e}
$$

em que: $y=$ vetor de dados; $r=$ vetor dos efeitos de repetição de cada época avaliada (efeitos fixos); $g$ = vetor dos efeitos genotípicos 
(efeitos aleatórios); $p=$ vetor dos efeitos de parcela (efeitos aleatórios); $i=$ vetor dos efeitos da interação genótipo x época de avaliação (efeitos aleatórios); $e=$ vetor de erros (efeitos aleatórios). As letras X, Z, W e T representam as matrizes de incidência para os referidos efeitos.

A análise de deviance foi utilizada a fim de testar a significância dos efeitos do modelo, empregando-se o teste da razão de verossimilhança (LTR), cuja significância foi testada pelo teste de qui-quadrado.

Nas estimativas do grau de relação entre as variáveis, calculou-se o coeficiente de correlação utilizando o teste $t$ para validar a significância. O progresso genético foi predito considerado os ganhos diretos, indiretos (resposta correlacionada) e índices de seleção. Adotaram-se como ganho desejado e pesos econômicos os equivalentes a um coeficiente de variação genético $(\mathrm{CVg})$ e um desvio-padrão genético (DPg), respectivamente.
A análise de divergência genética foi determinada utilizando a distância generalizada de Mahalanobis e, a partir da matriz de distância, obteve-se o agrupamento hierárquico pelo método UPGMA (Unweighted Pair Group Method using Arithmetic averages). O ponto de corte foi estabelecido por meio da metodologia de Mojena (1977) e o coeficiente de correlação cofenética foi adquirido de 1.000 permutações com significância testada pelo teste $t$.

\section{RESULTADOS}

Os efeitos aleatórios foram avaliados e se observou significância genotípica para os descritores morfológicos nas deviance individuais (Tabela 2). Essa significância evidenciou presença de variabilidade genética em cinco caracteres (NTR, CECP, CERP, CERI e \%RS), quando considerado os diferentes meses de avaliação (Tabela 2).

Tabela 2. Deviance individuais e conjunta e parâmetros genéticos em caracteres morfológicos em genótipos juvenis de pinhão-manso. Table 2. Individual and joint deviance and genetic parameters in morphological traits in juvenile physic nut genotypes.

\begin{tabular}{|c|c|c|c|c|c|c|c|c|}
\hline Efeitos & Meses & $\mathrm{AP}(\mathrm{cm})$ & DC $(\mathrm{cm})$ & NRT & CECP (mm) & CERP (mm) & CERI (mm) & RS (\%) \\
\hline \multirow{3}{*}{ Genótipo } & 3 & 0,01 & 0,01 & 0,01 & 0,30 & $2,86^{*}$ & 0,04 & - \\
\hline & 6 & 0,29 & 0,02 & $2,94 *$ & 1,58 & 7,39 *** & $11,66^{* * *}$ & $4,28 * *$ \\
\hline & 9 & 0,05 & 0,64 & 1,34 & $6,66^{* * *}$ & 1,25 & 1,22 & 1,52 \\
\hline \multirow{3}{*}{ Época } & 3 & 0,86 & 0,18 & 1,19 & 0,35 & 0,98 & 0,01 & - \\
\hline & 6 & 1,22 & 0,51 & 1,41 & $4,48^{* *}$ & 0,06 & 1,26 & 1,55 \\
\hline & 9 & 0,86 & 0,60 & $2,77 * *$ & $4,81 * *$ & 0,16 & 0,01 & $5,97 * *$ \\
\hline \multirow{3}{*}{ Média } & 3 & 74,84 & 35,77 & 2,52 & 1,85 & 2,43 & 2,15 & - \\
\hline & 6 & 136,57 & 57,53 & 10,81 & 1,62 & 1,84 & 1,79 & 23,69 \\
\hline & 9 & 184,16 & 74,02 & 20,91 & 1,61 & 1,82 & 1,82 & 0,56 \\
\hline Genótipo & & $14,26^{* * *}$ & $14,96 * * *$ & $20,54 * * *$ & $21,26 * * *$ & $35,87 * * *$ & $22,83^{* * *}$ & $16,54 * * *$ \\
\hline Época & & 0,45 & 0,04 & $4,37 * *$ & 1,86 & 2,06 & 1,14 & $10,04 * * *$ \\
\hline$G \times E$ & & 0,11 & 0,08 & $0,09 * *$ & 0,08 & 0,09 & 0,08 & $0,04 * * *$ \\
\hline Média & & 131,7 & 55,6 & 11,20 & 1,70 & 2,0 & 1,90 & 30,0 \\
\hline$h^{2} \mathrm{~m}$ & & 0,61 & 0,62 & 0,64 & 0,67 & 0,84 & 0,86 & 0,57 \\
\hline $\mathrm{CVg}(\%)$ & & 6,01 & 6,83 & 16,32 & 7,68 & 6,89 & 6,51 & 17,49 \\
\hline $\mathrm{CVr}$ & & 0,43 & 0,44 & 0,48 & 0,51 & 0,84 & 0,94 & 0,43 \\
\hline
\end{tabular}

AP: altura de planta; DC: diâmetro de caule; NRT: número de ramos totais; CECP: comprimento do entrenó caule principal; CERP: comprimento do entrenó de ramos primários; CERI: comprimento do entrenó de ramos produtivos; RS (\%): porcentagem de ramos secundários; $\mathrm{h}^{2} \mathrm{~m}$ : herdabilidade média de genótipo; CVg (\%): coeficiente de variação genética; CVr: coeficiente de variação relativa; ***, ** e * significativo pelo teste do qui-quadrado a 1,5 e 10 de probabilidade, respectivamente.

O efeito de época foi significativo para os caracteres NTR, CECP e \%RS aos seis e nove meses após o plantio, indicando influência do ambiente sobre o desempenho de, pelo menos, um genótipo para tais caracteres (Tabela 2).

A análise conjunta evidenciou efeito significativo da interação G x E para NRT e \%RS (Tabela 2), indicando que os genótipos apresentaram comportamento diferente ao longo das épocas de avaliações aos quais foram submetidos. Para esses caracteres, devem-se selecionar os genótipos mais promissores devido à ocorrência da interação. Nos demais caracteres, mesmo que as interações tenham sido não significativas, os efeitos genotípicos foram significativos (Tabela 2), demonstrando que há variabilidade genética entre os genótipos e sendo possível identificar quais genótipos possuem melhores médias para tais caracteres.

$\mathrm{A} \mathrm{h}^{2} \mathrm{~m}$ foi alta para os descritores, isto é, grande parte da manifestação fenotípica observada decorre de causas genéticas. A alta magnitude desse parâmetro genético pode indicar que o aumento no número de repetições (no tempo) tenha proporcionado esse resultado na análise conjunta. Logo, há uma maior confiabilidade na estimação dos valores genotípicos a partir dos valores fenotípicos observados.

$\mathrm{O} \mathrm{CVg}$ foi utilizado para comparar a variabilidade de cada caráter avaliado e inferir maiores diferenças genéticas dentro de cada tratamento. O $\mathrm{CVr}(\mathrm{CVg} / \mathrm{CVe})$ indicou predomínio dos componentes ambientais sobre os componentes fenotípicos (Tabela 2) em decorrência da juvenilidade dos genótipos avaliados.

Nos descritores agronômicos, devido sua maior importância agrícola, realizou-se uma análise REML a fim de identificar e estimar os componentes genéticos individuais que atuam como critérios de seleção. A variância fenotípica desses caracteres foi atribuída, principalmente, à variância ambiental, ou seja, houve influência do ambiente nas manifestações fenotípicas (Tabela 3). As variáveis NSF e M100 apresentaram efeito genotípico significativo, demonstrando variabilidade genética entre os tratamentos (Tabela 3). 
Tabela 3. Deviance individuais, componentes de variância e parâmetros genéticos em caracteres agronômicos em genótipos de pinhão-manso. Table 3. Individual deviance, components of variance and genetic parameters in agronomic traits in physic nut genotypes.

\begin{tabular}{lccccc}
\hline Efeitos & NFP & NSP & NSF & PROD $(\mathrm{g})$ & M100 $(\mathrm{g})$ \\
\hline Genótipo & 1,00 & 2,10 & $15,12^{* *}$ & 2,63 & $14,79 * *$ \\
Parcela & 0,53 & 0,18 & 1,34 & 0,37 & 0,01 \\
\hline$\sigma^{2}$ & 40521,67 & 307936,9 & 0,06 & 115859,5 & 59,92 \\
$\sigma_{\mathrm{f}}^{2}$ & 3071,09 & 28421,35 & 0,03 & 10708,3 & 19,78 \\
$\sigma^{2}$ parc & 3944,71 & 29294,97 & 0,01 & 14894,62 & 0,45 \\
$\mathrm{~h}^{2}$ & 0,31 & 0,38 & 0,76 & 0,35 & 0,74 \\
$\mathrm{r}_{\mathrm{g} \hat{g}}$ & 0,56 & 0,62 & 0,87 & 0,59 & 0,86 \\
$\mathrm{CVg}(\%)$ & 12,03 & 13,94 & 6,21 & 14,09 & 7,38 \\
CVr & 0,38 & 0,45 & 1,01 & 0,42 & 1,00 \\
Média & 460,79 & 1190,49 & 2,56 & 723,11 & 60,25 \\
\hline
\end{tabular}

NFP: número frutos/planta; NSP: número sementes/planta; NSF: número sementes/frutos; PROD: produção/planta; M100: massa de 100 sementes; $\sigma^{2} \mathrm{f}_{\text {: }}$ variância fenotípica individual; $\sigma_{\mathrm{g}}^{2}$ : variância genotípica; $\sigma^{2}$ parc: variância ambiental entre parcelas; $\mathrm{h}^{2}:$ herdabilidade no sentido amplo; $\mathrm{r}_{\mathrm{g} g}$ : acurácia; $\mathrm{CVg}(\%)$ : coeficiente de variação genética; CVr: coeficiente de variação relativa; ** e* significativo pelo teste do qui-quadrado a 1 e 5 de probabilidade, respectivamente.

A $h^{2}$ apresentou alta magnitude para NSF e M100, em que 76 e $74 \%$ da variação fenotípica observada foi decorrente de natureza genética, respectivamente (Tabela 3). A acurácia seletiva exibiu, consequentemente, alta magnitude para esses caracteres e moderada para os demais (Tabela 3).

O CVr esteve na unidade para NSF e M100 indicando que há genótipos favoráveis à seleção para esses caracteres, uma vez que a variância genotípica predominou na variância ambiental. Os genótipos de pinhão-manso apresentaram resultados promissores considerando que esta foi a primeira avaliação produtiva desses genótipos.

Nas estimativas das correlações genéticas, correlações significativas e positivas foram obtidas entre as variáveis, ou seja, os fatores genéticos contribuíram para a expressão desses caracteres (Tabela 4). Nos descritores morfológicos, as principais correlações envolveram as variáveis \%RS e NRT (Tabela 4).

Tabela 4. Correlação fenotípica entre caracteres morfológicos e agronômicos em genótipos de pinhão-manso.

Table 4. Phenotypic correlation between morphological and agronomic traits in physic nut genotypes.

\begin{tabular}{|c|c|c|c|c|c|c|c|c|c|c|}
\hline Caracteres & DC & NRT & CERP & CERI & $\mathrm{RS}(\%)$ & NFP & NSP & NSF & PROD & M100 \\
\hline $\mathrm{AP}$ & $0,41^{*}$ & $0,58^{* *}$ & $-0,23$ & $-0,22$ & $0,68 * *$ & $0,44^{*}$ & $0,40^{*}$ & 0,11 & 0,32 & $-0,20$ \\
\hline DC & - & $0,41 *$ & 0,14 & 0,13 & $0,43^{*}$ & $0,44^{*}$ & 0,3748 & $-0,11$ & 0,34 & 0,05 \\
\hline NRT & & - & $-0,31$ & $-0,30$ & $0,90 * *$ & $0,68^{* *}$ & $0,58^{* *}$ & 0,04 & $0,68 * *$ & 0,26 \\
\hline CERP & & & - & $0,99 * *$ & $-0,27$ & $-0,38^{*}$ & $-0,41 *$ & $-0,58 * *$ & $-0,31$ & $0,40^{*}$ \\
\hline CERI & & & & - & $-0,27$ & $-0,39 *$ & $-0,42 *$ & $-0,58^{* *}$ & $-0,33$ & $0,40^{*}$ \\
\hline $\mathrm{RS}(\%)$ & & & & & - & 0,63 & 0,55 & 0,11 & 0,59 & 0,11 \\
\hline $\mathrm{NFP}$ & & & & & & - & $0,97 * *$ & 0,36 & 0,92 & $-0,06$ \\
\hline NSP & & & & & & & - & 0,55 & $0,94 * *$ & $-0,10$ \\
\hline NSF & & & & & & & & - & $0,47^{*}$ & $-0,30$ \\
\hline PROD & & & & & & & & & - & 0,22 \\
\hline
\end{tabular}

AP: altura de planta; DC: diâmetro de caule; NRT: número de ramos totais; CERP: comprimento do entrenó de ramos primários; CERI: comprimento do entrenó de ramos produtivos; RS (\%): porcentagem de ramos secundários; NFP: número frutos/planta; NSP: número sementes/planta; NSF: número de sementes/frutos; PROD: produção/planta; M100: massa de 100 sementes; **, * significativo a 1 e $5 \%$ de probabilidade, respectivamente, pelo teste t.

Os caracteres agronômicos NFP e NSP se correlacionaram significativamente com a maioria dos caracteres, demonstrado que a seleção indireta será eficiente quando praticada nos descritores a eles correlacionados (Tabela 4). A variável PROD, um dos principais caracteres agronômicos, obteve correlações significativas entre NRT, NSP e NSF (Tabela 4).

Os caracteres CERP e CERI apresentaram correlações negativas e significativas com NFP, NSP e NSF (Tabela 4). Esses resultados ratificam o processo de avaliação precoce feito em plantas com crescimento vegetativo não cessado.

$\mathrm{Na}$ predição de ganhos genéticos, utilizou-se como critério de seleção as variáveis que apresentaram variância genética significativa e os caracteres correlacionados significativamente com o caráter produção. Os genótipos foram categorizados em ordem decrescente do sentindo de seleção e uma intensidade de seleção de 19\% foi aplicada.

Observou-se que a seleção direta proporcionou melhores ganhos que aos ganhos indiretos nos caracteres (Tabela 5). Os maiores progressos genéticos foram estimados para PROD e M100, contudo, para M100 a seleção direta derivou na redução dos demais caracteres morfoagronômicos em consequência do sentido e magnitude das correlações (Tabela 5). Nesse caso, recomenda-se utilizar os índices de seleção na predição dos ganhos genéticos.

Os índices de seleção também selecionaram plantas com maior PROD, M100 e NRT, tendo ganhos totais melhores que os da seleção direta (Tabela 5). O índice baseado em Soma de Ranks (MULAMBA; MOCK, 1978) obteve um melhor equilíbrio nas médias das variáveis, com melhor ganho total $(28,56 \%)$, seguido do Índice-Base (WILLIANS, 1962) com $23,46 \%$ e Distância Genótipo ao Ideótipo de 19,84\%. A partir desses resultados, a seleção simultânea foi mais eficiente que a seleção direta e indireta nos caracteres selecionados.

Após determinar os ganhos de seleção é necessário identificar os genótipos que deverão ser selecionados. Os genótipos que proporcionarão melhores progressos genéticos, considerando os índices Soma de Ranks e ÍndiceBase, são JCCE034, JCCE014 e JCCE103. Esses genótipos reúnem caracteres morfoagronômicos favoráveis presentes no germoplasma de J. curcas. 
Tabela 5. Progresso genético (\%) via seleção direta, indireta e índices de seleção em genótipos juvenis de pinhão-manso.

Table 5. Genetic progress (\%) by direct, indirect selection and selection indexs in juvenile physic nut genotypes.

\begin{tabular}{|c|c|c|c|c|c|}
\hline Caracteres principais & NRT & RS $(\%)$ & NSF & PROD & M100 \\
\hline & \multicolumn{5}{|c|}{ Ganhos de seleção diretos e indiretos } \\
\hline NRT & $5,49^{+}$ & 4,46 & 0,90 & 3,48 & $-0,78$ \\
\hline RS $(\%)$ & 4,10 & $4,69^{+}$ & 1,47 & 2,73 & $-0,91$ \\
\hline $\mathrm{NSF}$ & $-2,82$ & 1,09 & $4,59^{+}$ & 0,82 & $-1,38$ \\
\hline PROD & 5,63 & 6,48 & 4,43 & $11,68^{+}$ & $-0,26$ \\
\hline M100 & 6,53 & 1,44 & 0,70 & 4,75 & $9,63^{+}$ \\
\hline Índices de seleção & \multicolumn{5}{|c|}{ Ganhos de seleção via índices } \\
\hline Willians (1962) & 3,48 & 2,73 & 0,82 & 11,68 & 4,75 \\
\hline Mulamba \& Mock (1978) & 5,06 & 3,84 & 2,00 & 10,88 & 6,78 \\
\hline Genótipo-Ideótipo & 3,38 & 2,89 & 0,78 & 8,38 & 4,41 \\
\hline
\end{tabular}

NRT: número de ramos totais; RS(\%): porcentagem de ramos secundários; NSF: número sementes/frutos; PROD: produção/planta; M100: massa de 100 sementes; ${ }^{+}$ganhos com a seleção direta.

$\mathrm{Na}$ análise de divergência genética, quatro grupos foram formados pelo método UPGMA com um ponto de corte de $30 \%$ (Figura 1). A maioria dos genótipos apresentou grande similaridade, denotada pela menor distância genética, formando o maior grupo (laranja). O segundo grupo foi constituído por nove genótipos e o terceiro e quarto grupo se destacaram por terem sido formados, apenas, por um genótipo cada (Figura 1). Os genótipos JCCE036 e JCCE86 apresentaram maior divergência genética em relação aos demais e, por conseguinte, não foram agrupados nos demais clusters (Figura 1). A correlação cofenética foi de 0,84 e esse valor expressou boa representatividade entre a matriz cofenética e matriz de dissimilaridade, ou seja, o dendrograma representou confiabilidade nos agrupamentos.

A partir da distância genética, os genótipos JCCE 014

e JCCE103, selecionados nos ganhos de seleção, foram distribuídos no mesmo grupo e o genótipo JCCE034 em outro grupo mais distante (Figura 1).

Considerando as análises realizadas nesse estudo, os genótipos JCCE034, JCCE014, JCCE103, JCCE036 e JCCE086 proporcionam ganhos genéticos desejáveis e são genótipos contrastantes nos caracteres avaliados. Esses genótipos apresentam boa capacidade de serem utilizados como genitores no melhoramento de $J$ curcas.

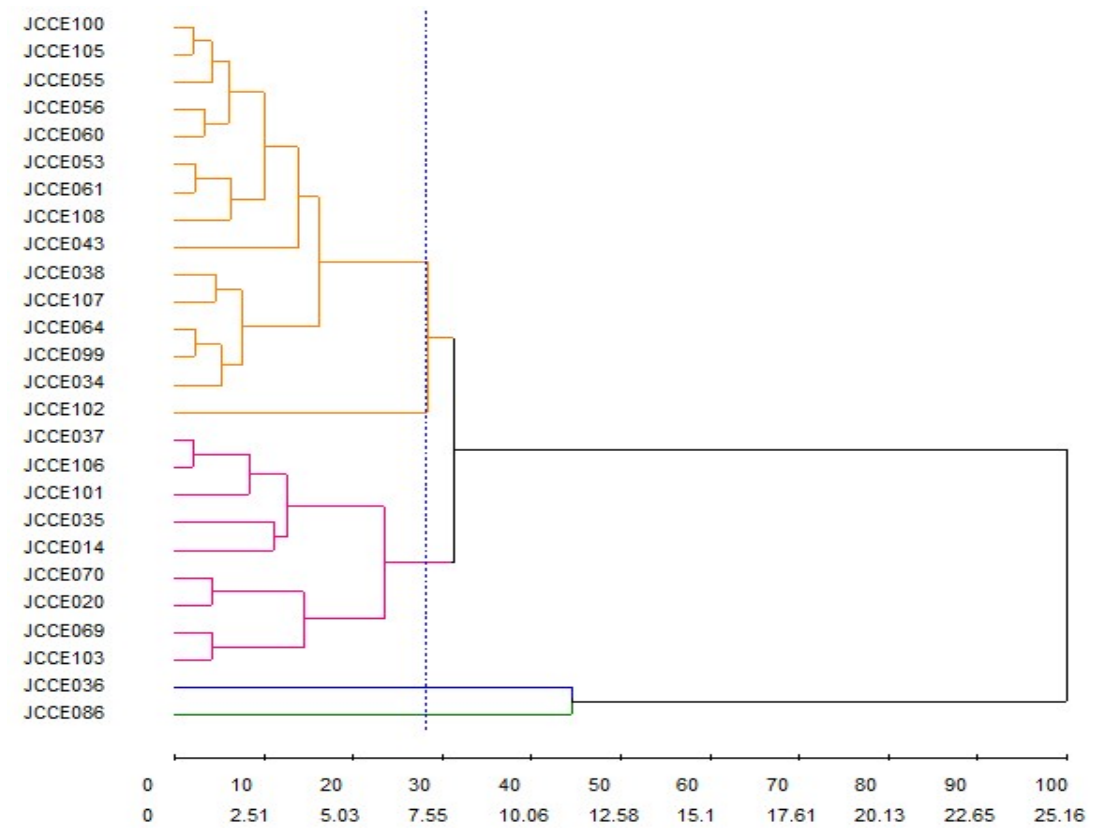

Figura 1. Dendrograma de dissimilaridade genética entre genótipos de pinhão-manso pelo método UPGMA. As cores representam os grupos formados e a linha tracejada corresponde ao ponto de corte.

Figure 1. Dendrogram of genetic dissimilarity among physic nut genotypes using the UPGMA method. The colors represent the clusters and the dashed line corresponds to the cutoff point.

\section{DISCUSSÃO}

Nas análises de deviance, individuais e conjunta, observouse que a maioria dos caracteres apresentaram variações fenotípicas atribuídas as variações ambientais. Contudo, considerando que as avaliações foram realizadas precocemente, no primeiro ano de ciclo da cultura, é possível observar que os genótipos de pinhão-manso obtiveram resultados promissores.
Morais et al. (2017) avaliaram a diversidade fenotípica de germoplasma brasileiro de pinhão-manso visando selecionar genótipos superiores para programas de melhoramento. Os caracteres morfológicos apresentaram altura de planta variando de 2,5 a $3,3 \mathrm{~m}$; diâmetro de caule de 12 a $31 \mathrm{~cm}$ e variação de dois a seis números de ramos primários.

$\mathrm{Na}$ avaliação agronômica, Peixoto et al. (2018), estudando famílias de meios-irmãos de pinhão-manso, encontraram 
média de 166,84 g de produção por planta e 64,26 g de massa de 100 sementes, no primeiro ano de ciclo produtivo. Os nossos resultados indicam que os caracteres morfológicos e de rendimento possuem bons efeitos de produção para o primeiro ciclo produtivo da cultura.

As avaliações morfológicas e agronômicas são importantes para o melhoramento vegetal por serem de fácil mensuração e obtenção. A partir dessas avaliações, os mecanismos genéticos com seus respectivos efeitos são estimados, constituindo ferramentas úteis na condução dos programas de melhoramento (RESENDE, 2007) e identificação de genótipos superiores no germoplasma.

Nas análises dos parâmetros genéticos, a herdabilidade ampla prediz os ganhos genéticos a serem obtidos, uma vez que é uma medida de proporcionalidade da variação fenotípica devida à natureza genética (RESENDE, 2007). Para os caracteres NSF e M100, a seleção se baseada no fenótipo do indivíduo permitirá a identificação acurada dos valores genéticos.

A acurácia seletiva, consequentemente, apresentou alta magnitude para essas variáveis. Esses resultados são indicativos de obtenção de progresso genético, uma vez que estão relacionados aos valores genotípicos verdadeiros do indivíduo, preditos a partir de informações extraídas dos ensaios (RESENDE, 2007).

Parâmetros genéticos foram preditos em famílias de meios-irmãos em pinhão-manso. Verificou-se variabilidade genética entre os genótipos com variância fenotípica atribuída à variância aditiva. $\mathrm{O} C \mathrm{Cr}$ para produção, esteve abaixo da unidade, sendo de 0,33 e massa de 100 sementes foi superior a unidade na análise REML (PEIXOTO et al., 2017) e 0,49 para produção e 0,86 para massa de 100 sementes na análise ANOVA, em avalições durante cinco anos de ciclo (PEIXOTO et al., 2018).

Correlações positivas e significativas foram observadas entre os caracteres, sobretudo, aos relacionados com a produção. Essas significâncias indicam que o aumento na produção de sementes de J. curcas está relacionado à seleção indireta de indivíduos vigorosos, com maiores números de ramos, frutos e massa de sementes.

Por outro lado, correlações negativas e significativas indicam associações negativas entre as variáveis, ou seja, o decréscimo do comprimento de entrenós nos ramos ocasiona aumento na produção de caracteres de rendimento, por estar relacionado à velocidade de crescimento da planta, demonstrando que os genótipos estão em pleno estágio de vigor.

Correlações genéticas foram obtidas para diferentes caracteres morfológicos e fisiológicos em 15 genótipos indianos de pinhão-manso (SINGH et al., 2016). O número de sementes por fruto obteve correlações positivas com o diâmetro dos ramos primários e correlações negativas com número de ramos primários e secundários (SINGH et al., 2016). Ayizannon et al. (2017) também encontraram correlações positivas e negativas entre caracteres morfoagronômicos de híbridos de J. curcas. Os resultados das correlações podem direcionar os resultados dos próximos cruzamentos.

Obter informações sobre caracteres morfológicos correlacionados com caracteres agronômicos favorece o processo de seleção indireta. Com essas associações, as estratégias de seleção serão planejadas usando apenas caracteres passíveis de seleção (CRUZ et al., 2012) e tornando essa etapa menos dispendiosa no melhoramento da espécie.
$\mathrm{Na}$ obtenção de ganhos genéticos, os índices de seleção estimaram os maiores progressos genéticos, na próxima geração, se comparado com a seleção direta, pois esta reduziu os ganhos de seleção para alguns caracteres de interesse. Nesse caso, três índices de seleção foram aplicados considerando o ganho genético desejado e distribuição dos pesos econômicos.

O Índice-base de Willians (1962) propõe a combinação linear dos valores fenotípicos, ponderado pelos seus pesos econômicos respectivos. O índice de Mulamba; Mock (1978), baseia-se na soma de postos (ranks) e consiste em classificar os genótipos em ordem crescente e favorável ao melhoramento. O índice Genótipo-ideótipo estima as distâncias ótimas entre os genótipos e valores de referências, determinados pelos máximos e mínimos observados.

Ganhos genéticos foram estimados em acessos de pinhão-manso, em que o número de ramos apresentou um ganho de $39,36 \%$ e o peso e rendimento de semente de 14,84 e 143,08\%, respectivamente (FRANCIS et al., 2017). Peixoto et al. (2018) também estimaram ganhos de seleção variando de $16,6-20,5 \%$ para produção e $1,9-5,2 \%$ para massa de 100 sementes, utilizando uma intensidade de seleção de $11 \%$ em famílias de meios-irmãos. Os resultados dos ganhos com a seleção variam entre os trabalhos, uma vez que são dependentes dos objetivos do melhorista e da intensidade de seleção aplicada. Portanto, deve-se requerer o alcance do ganho esperado sem exaurir a variabilidade genética dos genótipos.

A análise de agrupamento foi estabelecida pelo método UPGMA por permitir que as distâncias dentro dos clusters fossem visualizadas, oferecendo uma exposição mais detalhada da dissimilaridade genética entre os genótipos de pinhão-manso. Esse método de agrupamento originou um resultado mais preciso e sensato de acordo com o histórico dos genótipos, sendo o método escolhido.

Os genótipos apresentaram menores distâncias genéticas e dois grandes grupos foram formados considerando o ponto de corte determinado. Em contraste, dois genótipos de pinhão-manso provenientes do Ceará e Paraíba formaram clusters individuais. Esses genótipos, por apresentarem caracteres de interesse, podem ser utilizados como genitores contrastantes no processo de obtenção de progênies a serem melhoradas.

Os resultados ratificam similaridades genéticas em $J$. curcas, como observado por Ayizannon et al. (2017) que atribuiu a baixa variação genética da espécie devido à realização de seleções simples em populações locais presentes na África e América. Días et al. (2017) avaliaram a diversidade genética de germoplasma brasileiro de J. curcas utilizando marcadores moleculares. Os agrupamentos não foram capazes de revelar à origem geográfica dos acessos oriundos de diferentes países, pelo método UPGMA, corroborando para baixa a moderada diversidade genética de pinhão-manso ao redor do mundo (DÍAS et al., 2017).

A avaliação de germoplasma visando identificar melhores genótipos baseados na distância genética constitui outra estratégia adotada nos programas de melhoramento. Realizar cruzamentos entre genótipos divergentes de interesse têm objetivo de ampliar a base genética e selecionar os genitores para os cruzamentos artificiais.

Os genótipos que são selecionados para o mesmo conjunto de caracteres, normalmente, são próximos geneticamente (OLIVEIRA et al., 2013), contudo, se o cruzamento for realizado entre genótipos mais distantes 
haverá aumento na variabilidade nos próximos ciclos de seleção. Híbridos heteróticos também podem ser obtidos com esses cruzamentos e fixados na população otimizando a condução dos ensaios (CARDOSO et al., 2018).

Considerando as análises de progresso genético e seleção precoce realizadas nesse estudo, os genótipos JCCE034, JCCE014 e JCCE103 proporcionaram os melhores ganhos genéticos e os genótipos JCCE036 e JCCE086 foram os mais contrastantes na análise de dissimilaridade. Portanto, recomendam-se os cruzamentos entre esses cinco genótipos, por apresentarem boa capacidade de uso como genitores no melhoramento de J. curcas.

\section{CONCLUSÕES}

Os genótipos de pinhão-manso são promissores na seleção precoce, com base na predição das correlações e parâmetros genéticos.

Os progressos genéticos com uso de índices são mais favoráveis na seleção dos genótipos que utilizando seleções direta e indireta.

Três genótipos apresentam bom desempenho agronômico, com capacidade de compor grupos de genitores em cruzamentos direcionados no programa de J. curcas.

\section{AGRADECIMENTOS}

Os autores agradecem à Petrobras (Projeto PetroBio Girassol, subprojeto IV: Avaliação de recursos genéticos de Jatropha curcas) pelo apoio financeiro.

\section{REFERÊNCIAS}

ALVES, R. S.; TEODORO, P. E.; PEIXOTO, L. de A.; SILVA, L. A.; LAVIOLA, B. G.; DE RESENDE, M. D. V.; BHERING, L. L. Multiple-trait BLUP in longitudinal data analysis on Jatropha curcas breeding for bioenergy. Industrial Crops and Products, v. 130, p. 558-561, 2019. https://doi.org/10.1016/j.indcrop.2018.12.019

AQUINO, A. B.; AQUINO, B. F.; HERNANDEZ, F. F. F.; HOLANDA, F. J. M.; FREIRE, J. M.; CRISÓSTOMO, L. A.; COSTA, R. I.; UCHÔA, S. C. P.; FERNANDES, V. L. B. Recomendações de adubação e calagem para o Estado do Ceará. 1 ed. Fortaleza: UFC, 1993. 248p.

AYIZANNON, R. G.; AHOTON, L. E.; EZIN, V.; QUENUM, F.; MERGEAI, G. Improvement of physic nut (Jatropha curcas L.) by intraspecific hybridization between ecotypes of Africa and Americana. Journal of Plant Breeding and Crop Science, v. 9, p. 54-62, 2017. DOI: https://doi.org/10.5897/JPBCS2016.0620

CARDOSO, P. M. R.; DIAS, L. A. D. S.; RESENDE, M. D. V. D.; FREITAS, R. G. D.; CORREAA, T. R.; MUNIZ, D. R.; ZAIDAN, I. R. Genetic evaluation and selection in Jatropha curcas L. Crop Breeding and Applied Biotechnology, v. 18, n. 2, p. 192-199, 2018. DOI: http://dx.doi.org/10.1590/1984-70332018v18n2a27

CRUZ C. D.; REGAZZI A. J.; CARNEIRO P. C. S. Modelos Biométricos aplicados ao Melhoramento Genético. 4 ed. Viçosa: UFV, 2012. 514p.

DÍAZ, B. G.; ARGOLlO, D. M.; FRANCO, M. C.; NUCCI, S. M.; SIQUEIRA, W. J.; DE LAAT, D. M.; COLOMBO, C. A. High genetic diversity of Jatropha curcas assessed by ISSR. Genetics and Molecular Research, v. 16, n. 2, p. 1-12, 2017. DOI: http://dx.doi.org/10.4238/gmr16029683

FRANCIS, G.; OLIVER, J.; MULPURI, J. High yielding and trait specific genotypes and genetic associations among yield and yield contributing traits in Jatropha curcas L. Agroforestry Systems, v. 92, p. 1417-1436, 2017. DOI: http://dx.doi.org/10.1007/s10457-017-0089-2

LAVIOLA, B. G.; RODRIGUES, E. V.; RIBEIRO, L. P.; SILVA, L. A.; PEIXOTO, L. de A.; BHERING, L. L. Strategies in the Genetic Breeding of Jatropha curcas for Biofuel Production in Brazil. In: Mulpuri S., Carels N., Bahadur B. (Eds) Jatropha, Challenges for a New Energy Crop. Singapore: Springer, 2019. p. 45-62.

MAVUSO, C.; WU, Y. P.; CHEN, F. C.; HUANG, B. H. LIN, S. J. Genetic diversity analysis of Jatropha curcas L. accessions cultivated in Taiwan using inter simple sequence repeats (ISSR) markers. Agroforestry Systems, v. 90 , n. 3, p. 417-431, 2016. DOI: http://dx.doi.org/10.1007/s10457-015-9864-0

MOJENA, R. Hierarchical Grouping Methods and Stopping Rules: An Evaluation. The Computer Journal, v. 20, n. 4, p. 359-363, 1977 . DOI: https://dx.doi.org/10.1093/comjnl/20.4.359

MORAES, A. C. P. de; SILVA, F. M. da; CORRÊA, A. A. P.; MAURO, A. O. di; UNÊDA-TREVISOLI, S. H. Phenotypic diversity in physic nut (Jatropha curcas L.) in vivo germplasm bank for superior parent selection. African Journal of Biotechnology, v. 16, n. 17, p. 953961, 2017. http://dx.doi.org/10.5897/AJB2017.15890

MULAMBA, N. N.; MOCK, J. J. Improvement of potential of the Eto Blanco maize (Zea mays L.) population by breeding for plant traits. Egyptian Journal Genetics and Cytology, v. 7, p. 40-51, 1978.

OLIVEIRA, V. D.; RABBANI, A. R. C.; SILVA, A. V. C. D.; LÉDO, A. D. S. Genetic variability in physic nuts cultivated in Northeastern Brazil. Ciência Rural, v. 43, n. 6, p. 978-984, 2013. DOI: https://doi.org/10.1590/S0103-84782013005000060

PEIXOTO, L. A.; LAVIOLA, B. G.; ALVES, A. A.; ROSADO, T. B.; BHERING, L. L. Breeding Jatropha curcas by genomic selection: A pilot assessment of the accuracy of predictive models. Plos One, v. 12, p. 1-16, 2017. https://doi.org/10.1371/journal.pone.0173368

PEIXOTO, L. A.; TEODORO, P. E.; SILVA, L. A.; RODRIGUES, E. V.; LAVIOLA, B. G.; BHERING, L. L. Jatropha half-sib family selection with high adaptability and genotypic stability. Plos One, v. 13, n. 7, p. 1-19, 2018. https://doi.org/10.1371/journal.pone.0199880

RESENDE, M. D. V. Matemática e estatística na análise de experimentos e no melhoramento genético. 1 ed. Colombo: Embrapa Florestas, 2007. 561p.

SINGH, S.; PRAKASH, A.; CHAKRABORTY, N. R.; WHEELER, C.; AGARWAL, P. K.; GHOSH, A. Genetic variability, character association and divergence studies in Jatropha curcas for improvement in oil yield. Trees, v. 30, n. 4, p. 1163-1180, 2016. DOI: https://doi.org/10.1007/s00468-016-1354-0

SOUZA, R. D. L.; DIAS, L. A. D. S.; CORRÊA, T. R.; CAIXETA, E. T.; FERNANDES, E. D. C.; MUNIZ, D. R.; ROSMANINHO, L. B. C.; CARDOSO, P. M. R. Genetic variability revealed by microsatellite markers in a germplasm collection of Jatropha curcas L. in Brazil: an important plant for biofuels. Crop Breeding and Applied Biotechnology, v. 19, n. 3, p. 337-346, 2019. DOI: http://dx.doi.org/10.1590/198470332019v19n3a46

WILLIANS, J. S. The evaluation of a selection index. Biometrics, v. 18, p. 375-393, 1962. 